\title{
Aplicación de las funciones elípticas de Fourier para la descripción de la forma de los huevos de las aves
}

\author{
Dennis Denis Ávila \\ Dpto. Biología Animal y Humana, Facultad de Biología, Universidad de La Habana, calle 25 entre J e I, Vedado, \\ Cuba;dda@fbio.uh.cu
}

Recibido 14-I-2014. Corregido 27-VI-2014. Aceptado 29-VII-2014.

\begin{abstract}
Application of the elliptic Fourier functions to the description of avian egg shape. Egg shape is difficult to quantify due to the lack of an exact formula to describe its geometry. Here I described a simple algorithm to characterize and compare egg shapes using Fourier functions. These functions can delineate any closed contour and had been previously applied to describe several biological objects. I described, step by step, the process of data acquisition, processing and the use of the SHAPE software to extract function coefficients in a study case. I compared egg shapes in three birds' species representing different reproductive strategies: Cuban Parakeet (Aratinga euops), Royal Tern (Thalasseus maximus) and Cuban Blackbird (Dives atroviolaceus). Using 73 digital pictures of eggs kept in Cuban scientific collections, I calculated Fourier descriptors with 4, 6, 8, 16 and 20 harmonics. Descriptors were reduced by a Principal Component Analysis and the scores of the eigenvalues that account for $90 \%$ of variance were used in a Lineal Discriminant Function to analyze the possibility to differentiate eggs according to its shapes. Using four harmonics, the first five component accounted for $97 \%$ of shape variances; more harmonics diluted the variance increasing to eight the number of components needed to explain most of the variation. Convex polygons in the discriminant space showed a clear separation between species, allowing trustful discrimination (classification errors between 7-15\%). Misclassifications were related to specific egg shape variability between species. In the study case, A. euops eggs were perfectly classified, but for the other species, errors ranged from 5 to $29 \%$ of misclassifications, in relation to the numbers or harmonics and components used. The proposed algorithm, despite its apparent mathematical complexity, showed many advantages to describe eggs shape allowing a deeper understanding of factors related to this variable. Rev. Biol. Trop. 62 (4): 1469-1480. Epub 2014 December 01.
\end{abstract}

Key words: birds egg shape, Fourier descriptors, EFD, SHAPE software.

En los estudios sobre aves se ha brindado especial atención al huevo (Gill, 1998), por su papel central en la reproducción y la ecología del grupo. Los huevos poseen una amplia diversidad funcional, reflejada en los complejos patrones de tallas, formas y coloraciones que poseen. Las variaciones en el tamaño de los huevos han sido estudiadas con frecuencia por su relación con la estrategia reproductiva y su directa repercusión sobre las características de los pichones y el éxito reproductivo (Coulson, Potts, \& Horobin, 1969; Ricklefs, 1984; Jover,
Ruiz, \& González-Martín, 1993; Carey, 1996; Janiga, 1997; Martin et al., 2006). Sin embargo, los estudios oológicos históricamente se han enfocado hacia la medición de las dimensiones externas y del grosor de la cáscara, subestimándose la cuantificación de las formas y de los patrones de coloración.

En particular, la forma de los huevos de las aves no ha sido usualmente cuantificada, sino que se ha reducido a descripciones narrativas y comparativas. Probablemente, la forma del huevo es el resultado de la selección natural 
(Hoyt, 1976) y la variabilidad de las formas pudiera ser adaptativa, dada su importancia en la incubación (Orr, 1945; Lack, 1947).

La forma del huevo parece responder a numerosos efectos entre los cuales el más directo sería las tensiones de las paredes del conducto reproductor durante la puesta (Grant, 1982). Sin embargo, la forma también tiene un componente funcional que involucra un compromiso entre el volumen necesario para cada especie, el tamaño de la nidada y la capacidad o área de incubación de los adultos (Svensson, 1978; Ojanen, Orell, \& Vaisinen, 1979; Otto, 1979; Gill, 1998). Formas redondeadas presentan mayor relación superficie/volumen, $\mathrm{y}$ son características de aves de gran tamaño y/o robustas (como por ejemplo las Galliformes), por tanto, aunque el tamaño de la nidada sea grande, el volumen del huevo respecto al de los adultos es mucho menor. Los huevos con forma alargada o semicónica son característicos de aves relativamente pequeñas; o sea, que ponen huevos relativamente más grandes y además, numerosos (como por ejemplo algunos Charadriiformes) (Gill, 1998).

El estudio de la variación en las formas de los huevos dentro de una nidada ha mostrado la existencia de patrones como, por ejemplo, que el diámetro mayor y el radio de curvatura del polo mayor, así como la asimetría del huevo, tienden a ser más pequeños en el último huevo de la nidada (Preston \& Preston, 1953). En ese caso, las diferencias en la forma deben reflejar las adaptaciones a condiciones medioambientales diferentes o quizás un compromiso entre las ventajas estructurales, el volumen de puesta, el tamaño y el contenido del huevo. Por ejemplo, los huevos con forma esférica maximizan la conservación del calor, la resistencia de la cáscara y el ahorro de los materiales en la formación de la misma (Barta \& Székely, 1997; Gill, 1998). También se han mencionado, posibles factores ecológicos selectivos relacionados con el hábitat de nidificación y las probabilidades de caída del nido que influyen sobre sus formas. Los huevos más puntiagudos tienden a rodar menos, en un arco menor que los huevos que tienen forma redondeada, disminuyendo así la probabilidad de caer o alejarse del nido.

El supuesto desinterés actual al estudio de las formas de los huevos, posiblemente tiene su base en la dificultad práctica de su cuantificación. A pesar de ser objetos biológicos de formas relativamente simples, la forma de un huevo es muy difícil de describir o cuantificar numéricamente. Se han seguido varios enfoques matemáticos en algunos trabajos clásicos, pero que no tuvieron mucho eco en investigaciones posteriores (Preston, 1953; Gemperle \& Preston, 1955; Preston, 1968; Barta \& Székely, 1997). Estos y otros estudios utilizaron las dimensiones lineales y sus proporciones como indicadores de la forma, pero esto puede distorsionar o enmascarar las diferencias, ya que el propio concepto de "forma", excluye el tamaño.

A partir de la última década del pasado siglo, se ha ido consolidando un nuevo paradigma que enfatiza la "captura" y análisis de la forma de una manera integral y no dependiente de las dimensiones, que se denomina Morfometría geométrica (Adams, Rohlf, \& Slice, 2004). Este enfoque se basa en la digitalización de contornos o puntos clave, cuyas conformaciones espaciales son analizadas matemáticamente en el contexto de la teoría de los espacios de forma (Kendall, 1977). Dentro de este grupo de nuevas técnicas, los métodos de contornos se basan en la digitalización de los puntos alrededor de un contorno y ajustarlo a una función matemática, usualmente derivada de los análisis de Fourier, para emplear en estudios comparativos los coeficientes de estas como variables multidimensionales derivadas.

Los descriptores elípticos de Fourier (DEF), propuestos por Kuhl y Giardina (1982), pueden delinear cualquier tipo de forma con un contorno cerrado bidimensional y ya han sido aplicados al análisis de varias formas biológicas (Kincaid \& Schneider, 1983; Rohlf \& Archie, 1984; Furuta, Ninomiya, Takahashi, Ohmori, \& Ukai, 1995; Andrade, Mayo, Kirkup, \& Van den Berg, 2010; Zhan \& Wang, 2012). Más recientemente han sido empleados como herramientas para la detección de 
patrones y la identificación automatizada de especies (Neto, Meyer, Jones, \& Samal, 2006; Kim, Kim, \& Kim, 2011; Singh, Gupta, \& Gupta, 2013). Sin embargo, no son conocidos trabajos que empleen este método en el estudio de las formas de los huevos de las aves (excepto Denis \& Olavarrieta, 2011). Por las razones antes expuestas, en el presente trabajo se describe la aplicación de este método (DEF) en la caracterización y comparación de la forma de los huevos de las aves, tomando como estudio de caso tres especies con diferentes estrategias reproductivas: Aratinga euops (Wagler) (especie endémica de la familia Psittacidae, localmente conocida como Catey), Thalasseus maximus (Boddaert) (Gaviota Real, familia Laridae) y Dives atroviolaceus (d'Orbigny) (especie endémica de la familia Icteridae, de nombre común Totí). Este sencillo método puede contribuir a refinar los empleados históricamente en el análisis de la forma de los huevos a partir de dimensiones lineales y aumentar el espectro de posibilidades para el estudio de los patrones ecológicos y evolutivos en la reproducción de las aves.

\section{MATERIALES Y MÉTODOS}

Fundamento matemático: El análisis de los contornos se basa en la digitalización de la silueta de un objeto, que expresada como una secuencia de coordenadas $(\mathrm{x}, \mathrm{y})$ pueden ser manipuladas matemáticamente y ajustadas a una ecuación derivada de las funciones de Fourier. Jean Baptiste Joseph Fourier (1768-1830) demostró que cualquier función periódica se puede producir por combinaciones lineales de senos y cosenos con factores de multiplicación apropiados (amplitudes). Cada uno de estos senos o cosenos constituyentes de la función se denominan armónicos. Los contornos cerrados de formas simples, pueden expresarse en coordenadas polares con el radio como función del ángulo a partir de un punto interno fijo, lo cual constituye una función periódica. De esta manera, toda la información sobre la forma que se encontraba en la secuencia de puntos se reduce a un número menor de parámetros cuya distribución puede estudiarse en un espacio morfológico con los coeficientes como ejes. Sin embargo, este espacio aún es altamente dimensional por lo que generalmente es necesario reducirlo con alguna técnica multivariada, como el análisis de componentes principales (Rohlf \& Archie, 1984). Los puntajes de los vectores propios de estos análisis pueden usarse como "valores" de las características morfológicas en otros análisis.

Los descriptores elípticos de Fourier son una extensión de este método, aplicable cuando los contornos son tan complejos que pudiera existir más de un valor de radio por cada ángulo, y son los más populares de los análisis de formas de Fourier (Ferson, Rohlf, \& Koehn, 1985). El método se desarrolla tomando los incrementos en $\mathrm{X}$ y $\mathrm{Y}$ entre puntos, para definir la función periódica (Kuhl \& Giardina, 1982). Tiene el inconveniente de que produce el doble de parámetros que el análisis simple.

Si el contorno de una forma digitalizada se representa como una secuencia de puntos (p) ordenados y registrados en el sentido de las manecillas del reloj comenzando por un punto arbitrario, y las distancias entre puntos son lineales, entonces el segmento entre $(i-1)$ y el punto $\mathrm{p}$ es:

$$
\Delta t_{p}=\sum_{i=1}^{p} \Delta t_{i}
$$

y la sumatoria de todos estos incrementos sería el perímetro del contorno.

Entonces, la expansión elíptica de Fourier de la secuencia de coordenadas $\mathrm{X}$ es:

$$
x_{p}=x_{c e n}+\sum_{n=1}^{\infty}\left(a_{n} \cos \frac{2 n \pi t}{T}+b_{n} \operatorname{sen} \frac{2 n \pi t_{p}}{T}\right)
$$

donde

$$
a_{n}=\frac{T}{2 n^{2} \pi^{2}} \sum_{p=1}^{K} \frac{\Delta x_{p}}{\Delta t_{p}}\left(\cos \frac{2 n \pi t_{p}}{T}-\cos \frac{2 n \pi t_{p-1}}{T}\right)
$$
y

$$
b_{n}=\frac{T}{2 n^{2} \pi^{2}} \sum_{p=1}^{K} \frac{\Delta x_{p}}{\Delta t_{p}}\left(\operatorname{sen} \frac{2 n \pi t_{p}}{T}-\operatorname{sen} \frac{2 n \pi t_{p-1}}{T}\right)
$$


En estas ecuaciones, $x_{\text {cen }}$ es el coeficiente del punto central y $\mathrm{n}$ es el número armónico de coeficientes $\left(a_{n}\right.$ y $\left.b_{n}\right)$. Los coeficientes para las coordenadas $\mathrm{Y}\left(\mathrm{c}_{\mathrm{n}} \mathrm{y} \mathrm{d}_{\mathrm{n}}\right)$ se calculan de la misma forma. Estos coeficientes deben ser normalizados para hacerlos invariantes al tamaño, a la rotación y al punto inicial, de acuerdo al tamaño y orientación del eje mayor, del radio mayor o normalizando los radios.

El ajuste de estas funciones requiere de extensivos cálculos matemáticos, que actualmente son desarrollados por varios programas computarizados de análisis de datos como el SHAPE o el PAST.

Toma de datos: La toma de los datos para el análisis de un contorno parte de la digitalización del contorno de los objetos. En dependencia del programa que se use se generan puntos equiangulares por interpolación lineal entre las coordenadas que se proveen (programa PAST) o se extrae un código de cadena (programa SHAPE). Aunque puede lograrse con un tablero digitalizador, u otro sistema alternativo de entrada de datos digitales, lo más usual es emplear una imagen digital (fotografía o escaneo) del objeto a estudiar. En este estudio de caso, se empleó una muestra total de 78 fotografías de tres especies, representantes de tres estrategias ecológicas de cría de las más frecuentes: A. euops $(\mathrm{n}=19)$, como representante de aves que nidifican en cavidades; $D$. atroviolaceus $(\mathrm{n}=41)$, representante de un ave de bosque que cría en nidos construidos con ramillas por los parentales; y Thalasseus maximus $(\mathrm{n}=18)$, ave marina de reproducción colonial que pone los huevos en el suelo y tiene pichones nidífugos. Las imágenes fueron tomadas de la colección de huevos del Instituto de Ecología y Sistemática, con una cámara digital Canon Powershoot SX500, de 16Mp de resolución. Las fotografías se tomaron poniendo el huevo sobre un fondo de color contrastante, desde una ubicación perpendicular, a unos $20 \mathrm{~cm}$ de distancia, y en un lugar con luz difusa abundante y sin flash, para evitar la creación de sombras. Las imágenes se manipularon digitalmente en Adobe Photoshop v9.0, en el cual se eliminó el color de fondo y se aumentó el contraste de la imagen hasta que el huevo quedara totalmente en blanco y el fondo en negro, y se guardaron como bitmaps (extensión BMP) en formato RGB de 8bits.

Por las características de la forma de entrada de los datos, en el estudio de los huevos parece ser más apropiado el programa SHAPE v.1.3, diseñado para evaluar las formas de contornos basándose en transformadas elípticas de Fourier (Iwata \& Ukai, 2002). SHAPE contiene cuatro subprogramas llamados ChainCoder, Chc2Nef, PrinComp y PrinPrint para el procesamiento de las imágenes digitales, la obtención del código de cadena y de los coeficientes de Fourier, y el desarrollo de un Análisis de Componentes Principales. Incluye también rutinas para la visualización de las formas a partir de los datos ya digitalizados (ChcViewer y NefViewer).

El código de cadena es un sistema de codificación para describir la información espacial de los contornos con números del 0 al 7 (Freeman, 1974): los dígitos indican la dirección del próximo paso alrededor de un contorno: 0 $=$ un paso a la derecha, $2=$ un paso arriba, $4=$ uno a la izquierda, $6=$ uno abajo, y los otros dígitos son direcciones intermedias. Para obtener este código debe emplearse el subprograma ChainCoder con las imágenes de los huevos. Este subprograma lee las imágenes BMP, las convierte a escala de grises, las binariza a partir de un valor umbral seleccionado en el histograma de la imagen, elimina los posibles ruidos existentes en las imágenes por medio de filtros de erosión - dilución y obtiene el código de cadena, que queda guardado en un fichero ASCII con extensión .chc.

A partir del fichero del código de cadena, con el programa Chc2Nef, se calcularon los coeficientes de las transformadas de Fourier para 4, 5, 816 y 20 armónicos consecutivamente, utilizando como normalización la longitud y orientación del radio mayor. Los coeficientes de Fourier se almacenaron en un fichero ASCII de extensión .nef que fueron utilizados para el análisis multivariante posterior. 
Con la gran cantidad de variables producidas (cuatro coeficientes por cada armónico) (entre 16 y 80 valores por huevo) se realizaron Análisis de Componentes Principales (ACP), empleando las matrices de varianza - covarianza con lo cual se reduce la dimensionalidad, y se crean nuevas variables derivadas que ordenan a los individuos en un espacio canónico de forma y que son manipulables estadísticamente. Los puntajes de los componentes en cada ejemplar se tomaron para realizar Análisis de Funciones Discriminantes. Se estimó el número de armónicos suficientes para producir clasificaciones satisfactorias y para comprobar el efecto de la cantidad de componentes sobre el error de discriminación, con los resultados de los coeficientes obtenidos con 16 armónicos, se realizaron 7 discriminantes consecutivos, incorporando en cada análisis, un nuevo componente. Los componentes principales se obtuvieron con el subprograma PrinCom del SHAPE, y los análisis estadísticos se realizaron con el Statistica v8.0.

\section{RESULTADOS}

Independientemente del número de armónicos empleados para describir el contorno, el primer componente explicó cerca de la mitad de la variabilidad (entre 44 y 47\%) (Fig. 1). A medida que aumentó el número de armónicos empleados, se requirió mayor cantidad de componentes para explicar el $90 \%$ de la variación en la forma, por lo cual en dependencia del objetivo de los estudios se debe buscar un balance entre mayor resolución y menor cantidad de variables. De cualquier forma, con el resultado del primer componente, y al emplear la inversión de las transformadas de Fourier, se puede reconstruir el contorno de los huevos y caracterizar gráficamente su variabilidad (una de las ventajas de los métodos de morfometría geométrica) (Fig. 2).

Al realizar el Análisis de Función Discriminante con los cinco componentes del ACP realizado con cuatro armónicos $(90 \%$ de varianza explicada), se obtuvo que el primer

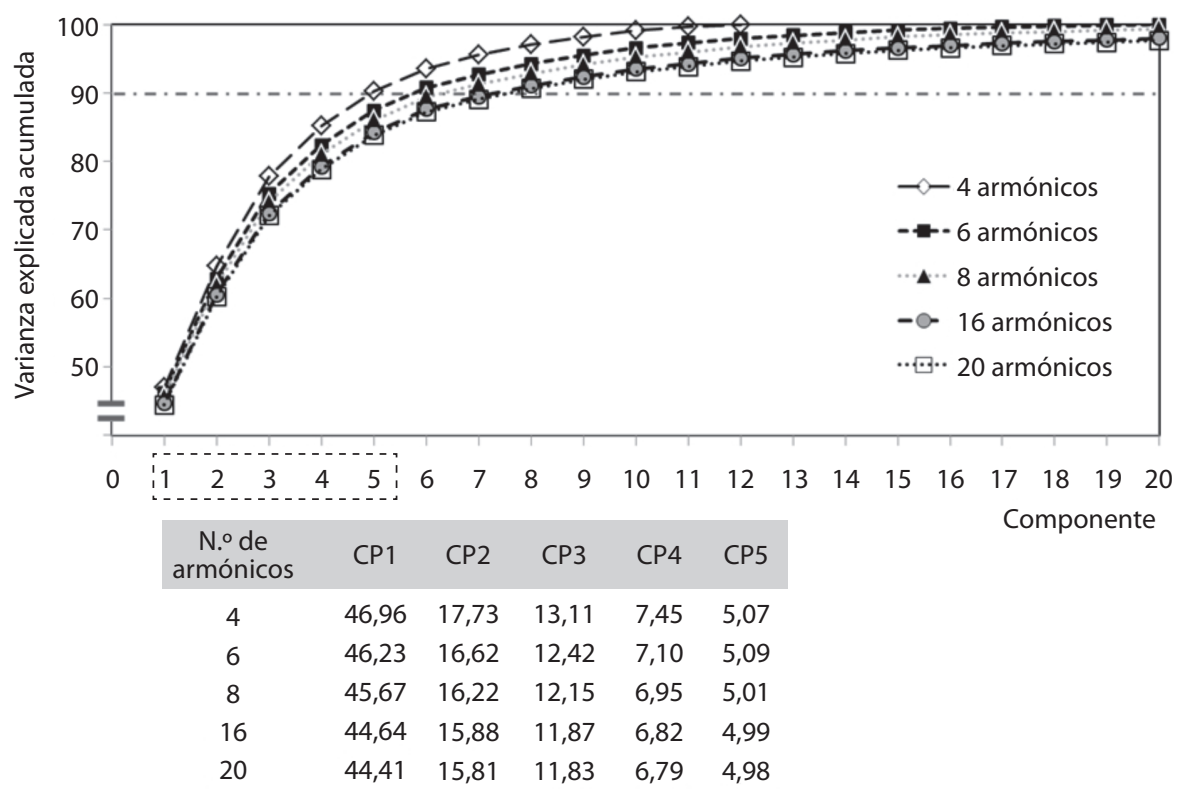

Fig. 1. Comportamiento de la varianza explicada por los componentes principales a medida que se incrementa el número de armónicos empleados para la caracterización de la forma del huevo en tres especies de aves (Catey, Totí y Gaviota Real). Fig. 1. Trend in the explained variance of the principal component with the increment of the number of harmonics used to characterize egg shape in three birds species (Cuban Parakeet, Cuban Blackbird and Royal Tern). 

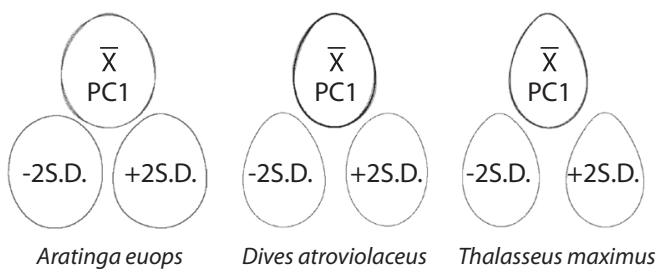

Fig. 2. Reconstrucción digital, a partir del primer componente principal obtenido con las transformadas elípticas de Fourier de los contornos, de la forma media de los huevos y sus variantes extremas en tres especies de aves.

Fig. 2. Digital reconstruction, using the main principal component of the Elliptic Fourier Descriptors of eggs outlines, of mean egg shapes and its extreme variations in three birds species.

autovalor explicó el $97.03 \%$ de las diferencias (Cuadro 1). Los huevos de A. euops fueron perfectamente diferenciables, pero en el Totí hubo un $27 \%$ de errores de clasificación. Solo un caso de huevo de Gaviota fue mal clasificado, confundido con uno de Totí. En general, el porcentaje de errores de clasificación en este caso fue de un $15.6 \%$.

Si en lugar de cuatro armónicos, se describe la silueta del huevo con seis armónicos, el $90 \%$ de la varianza es explicada por los primeros seis componentes. El discriminante llevado a cabo con estos obtiene una mejora notable, disminuyendo el error general de clasificación a 7.69\%. Al continuar empleando un número mayor de armónicos no mejoran los errores de clasificación, lo cual indica que ya con seis u ocho armónicos es suficiente para describir la forma de una figura simple como el huevo.

La capacidad de discriminar eficientemente también depende del número de componentes que se utilicen como variables, dado que cada uno de ellos explica una porción diferente de las diferencias en formas, al ser ortogonales entre sí. Esta relación, sin embargo no es completamente lineal y su comportamiento difiere entre especies (Fig. 3). De cualquier forma, es recomendable emplear siempre la cantidad de componentes que, en total, expliquen una porción de varianza aceptable.

El diagrama de ordenamiento de los ejes discriminantes para la forma de los huevos, para la descripción del contorno con 16 armónicos y empleando los primeros ocho componentes (que explican $91.1 \%$ de la varianza) se muestra en la figura 4. La separación de los polígonos convexos mínimos hace evidente la posibilidad de discriminar los huevos a partir de estas variables de forma. Los puntajes para el primer componente son poco variables en general $(\mathrm{CV}=1.61 \%)$, con diferencia entre especies. La gaviota mostró la menor

\section{CUADRO 1}

Análisis de Función Discriminante realizados con los puntajes de los ACP de los Coeficientes Elípticos de Fourier obtenidos a partir de un número creciente de armónicos sobre el contorno de los huevos de tres especies de aves

TABLE 1

Discriminant Function Analysis with PCA scores of the Elliptic Fourier Descriptors, obtained from a growing number of harmonics on eggs outlines in three birds species

\begin{tabular}{ccccccc}
$\begin{array}{c}\text { No. de } \\
\text { armónicos }\end{array}$ & Autovalor & $\begin{array}{c}\text { Varianza Explicada } \\
(\text { CP }>90 \%)^{*}\end{array}$ & Aratinga euops & Thalasseus maximus & Dives atroviolaceus & Total \\
4 & 2.97 & $97.03(5)$ & 0.0 & 5.56 & 27.50 & 15.58 \\
6 & 4.43 & $95.11(6)$ & 0.0 & 11.11 & 9.76 & 7.69 \\
8 & 4.53 & $93.19(7)$ & 0.0 & 5.56 & 12.20 & 7.69 \\
16 & 4.54 & $92.82(8)$ & 0.0 & 5.56 & 12.20 & 7.69 \\
20 & 5.02 & $93.40(8)$ & 0.0 & 5.56 & 12.20 & 7.69 \\
\hline
\end{tabular}

* Número de componentes principales que explican más del 90\% de la varianza. 


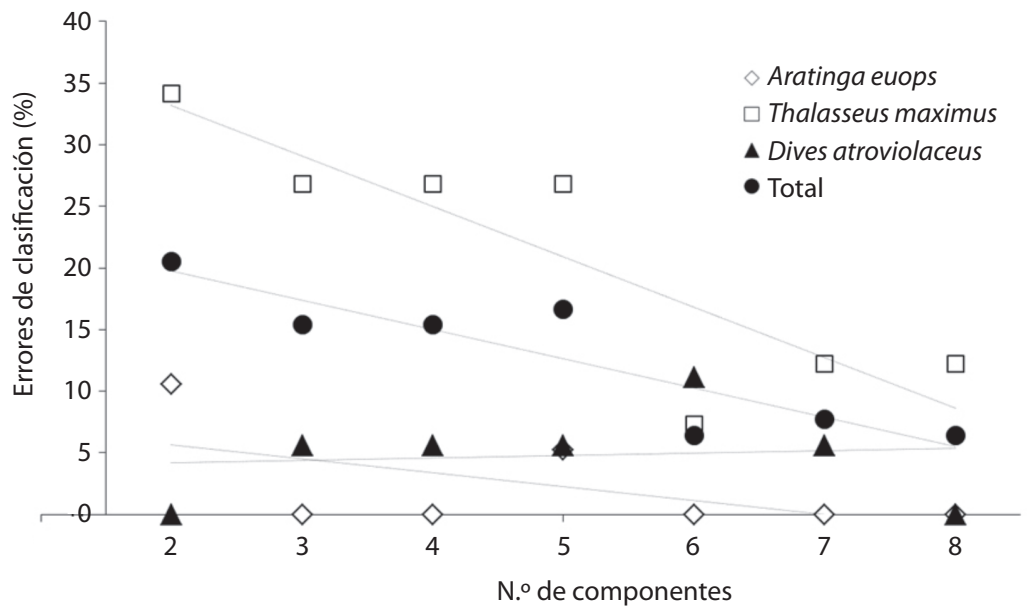

Fig. 3. Comportamiento del porcentaje de errores de clasificación a medida que se incorporan más variables explicativas (componentes) al análisis discriminante con las formas de los huevos en tres especies de aves.

Fig. 3. Trends of misclassification percentage while adding more explicative variables (components) to the Discriminant Function Analysis with eggs shape descriptors in three bird species.

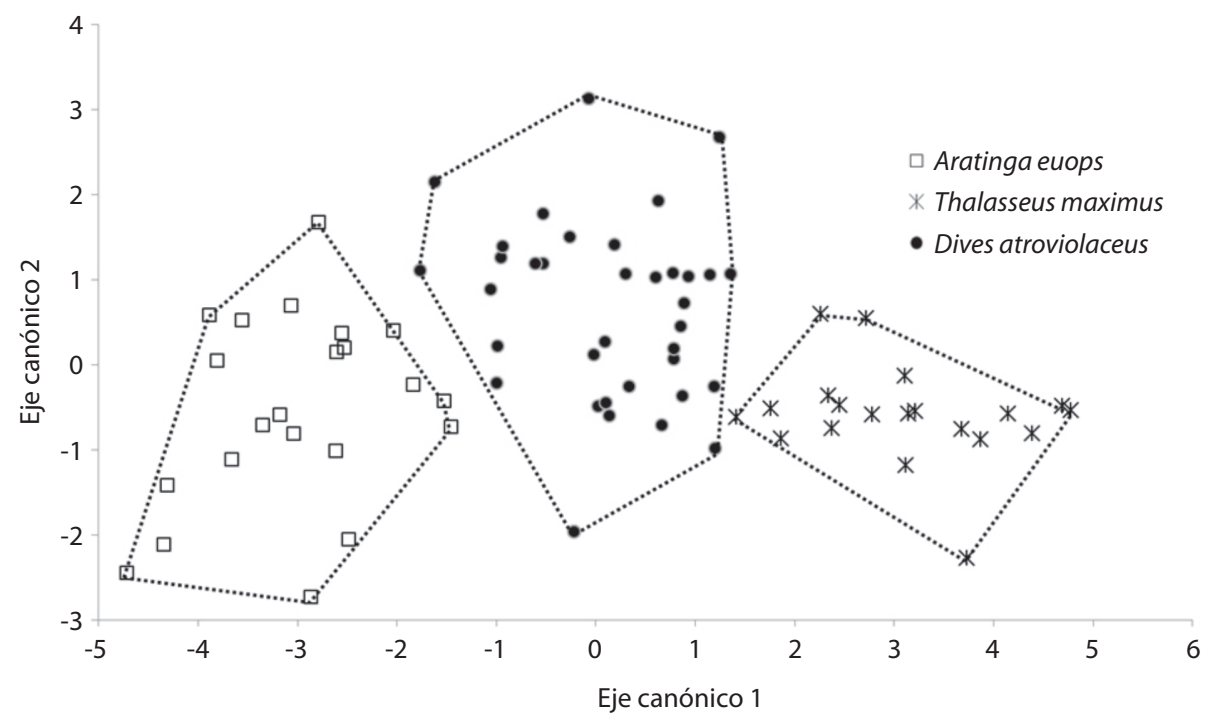

Fig. 4. Diagrama de ordenamiento del análisis discriminante realizado empleando como variables de forma de los huevos los componentes principales resultantes de la reducción dimensional de los coeficientes elípticos de Fourier de 16 armónicos en el contorno del huevo de tres especies de aves.

Fig. 4. Ordination plot of the Discriminant Analysis using as shape variables the principal component, to reduce dimensionality of elliptic Fourier coefficients for 16 harmonics in egg outlines in three bird species. 
variabilidad $(\mathrm{CV}=0.62 \%)$, seguida del psitácido $(\mathrm{CV}=0.77 \%)$ y del ictérido $(\mathrm{CV}=1.01 \%)$.

\section{DISCUSIÓN}

Debido a las tendencias históricas de las ciencias naturales hacia la cuantificación y al reduccionismo, los estudios morfométricos siempre se derivaron al estudio de las dimensiones. Las primeras aproximaciones al estudio de las formas se desarrollaron a partir de índices: relaciones entre dimensiones lineales que dan idea de las proporciones. Pero "proporción" no es equivalente a "forma".

El estudio de la forma de los huevos fue relegado a un lugar secundario por el análisis de las variaciones en los tamaños, dada su evidente y directa relación con las estrategias de la historia de vida de las aves. Sin embargo, se ha mostrado para varias especies que ambas variables tienen un fuerte componente genético (Vaisinen, Hilden, Soikkeli, \& Vuolanto, 1972; Kendeigh, 1975; Ojanen et al., 1979; van Noordwijk, Keizer, Van Balen, \& Scharloo, 1981). Una evidencia de la heredabilidad de estos componentes ha sido la repetibilidad de las formas y tamaños entre sucesivas nidadas de una misma hembra (Petersen, 1992). Ahora bien, aún no está clara la significación ecológica o evolutiva de estos patrones genéticos. Hoyt (1976) buscó su posible significado en la relación área-volumen, de probada significación en muchos sistemas biológicos. Según Grant (1982), si la forma es adaptativa, su significación debe relacionarse con la incubación y las proporciones corporales de los padres (Orr, 1945; Lack, 1947).

También existe la posibilidad de que no sea un carácter primario adaptativo, sino un reflejo de las características corporales de la madre y en particular de su talla, y que su patrón sea un subproducto. La máxima distensibilidad del oviducto debe constituir un límite para el ancho de los huevos, cuyo incremento en volumen, solo sería posible con una elongación superior (van Noordwijk et al., 1981). Por esta razón, es esperable la ausencia de correlaciones entre ambas dimensiones y que la variabilidad del ancho sea menor que la del largo y su correlación con el peso corporal mucho mayor, lo cual ha sido verificado en algunas especies (Grant, 1982).

En su trabajo clásico sobre la forma de los huevos, Preston (1968) mencionaba que las formas de los huevos podían ser descritas por tres parámetros: elongación, asimetría y bicono, que resumían distintos aspectos de la forma sin estar correlacionados entre sí. De estas, el más utilizado ha sido el índice de elongación dado como la simple razón entre los diámetros mayores y menores.

Un notable trabajo relacionado con el estudio de la forma de los huevos es el de Petersen (1992), quien registra la forma de 1743 huevos provenientes de más de 300 nidos de Ganso Emperador (Chen canagicus), para evaluar su utilidad como carácter identificador del parental, al considerarla controlada genéticamente y altamente constante dentro de cada individuo. A partir de fotografías analógicas sobre una rejilla calculó para cada huevo 18 índices: siete propuestos por Mand, Nigul, y Sein (1986), cinco propuestos por Preston (1968), y seis por Preston (1974). Estas variables las redujo por medio de un análisis de componentes principales, tomando los vectores de mayor variación para un análisis de agrupamiento. La mayor varianza fue aportada por aquellos índices que dependían de la talla (volumen, área transversa y superficie) (31\%) y en segundo lugar por variables que describían las características de los extremos del huevo (redondeo o conicidad) (30.4\% de varianza). A pesar de que el análisis estadístico que emplea no ofrece alta seguridad para clasificar los individuos, obtuvo bajos porcentajes de errores: $77.8 \%$ de los huevos clasificados como similares eran realmente de la misma nidada, pero los errores por comisión fueron también elevados (del 46\%).

Una de las posibles desventajas del empleo de estos índices se relaciona con los problemas de potencia que pueden presentar en los análisis estadísticos. Grant (1982) describe la variación en forma de los huevos entre 10 especies de pinzones de Darwin, aproximándola a elipses perfectas y asumiendo que los "coeficientes 
de forma", que modularían la diferencia con la figura geométrica, no sesgarían notablemente los resultados. Este autor caracteriza la forma analizando la pendiente de la relación lineal entre los diámetros mayor y menor pero, en general, no encontró diferencias entre especies. Briskie y Sealy (1990) describiendo la forma de los huevos de Empidonax minimus (Bobito Menor, familia Tyrannidae) con el índice de elongación tampoco encontraron diferencias con el orden de puesta, con el tamaño de nidada ni con el año.

Sin embargo, también puede aparecer lo contrario: Denis (2002) hizo un análisis del índice de elongación de los huevos de siete especies de garzas (Ardeidae) y detectó diferencias altamente significativas entre todas las especies. Sin embargo, empleando los DEF y ocho puntos clave equiangulares como landmarks no encontró tales diferencias una vez que se eliminó el efecto de las diferencias afines (rotación, traslación y escalado isotrópico) (Denis \& Olavarrieta, 2011). En el primer estudio, posiblemente el resultado fuese un artificio estadístico por el elevado tamaño de muestra que se empleó ( $\mathrm{n}$ total= 1748 huevos) que llevó a detectar diferencias en tamaños de efecto de pocos milímetros de diferencia, de significación biológica poco probable.

Los DEF proporcionan una vía cuantitativa elegante para describir los patrones de variación en formas a partir de siluetas cerradas (McLellan \& Endler, 1998) y pueden detectar diferencias sutiles en formas entre individuos y poblaciones (White \& Prentice, 1988; Lonn \& Prentice, 1995). Estudios genéticos han mostrado que la variación en forma de las hojas de algunas plantas (como los cítricos), detectada por estos coeficientes, tiene un componente hereditario significativo (Iwata, Nesumi, Ninomiya, Takano, \& Ukai, 2002).

Las tres especies de aves tomadas como ejemplo pertenecen a grupos ecológica y taxonómicamente muy diferentes, por lo cual era de esperar que se detectaran diferencias en la forma del huevo. Si la forma del huevo - su elongación y asimetría- se relaciona con el riesgo de caída de los nidos, es evidente que en caso del psitácido al nidificar en cavidades de árboles, no tiene sentido una forma alejada de la esfera. Esta forma, además, maximiza la relación área volumen, con lo cual se optimiza el intercambio de gases, que puede llegar a ser más crítico en estas cavidades donde se ha registrado una hipoxia significativa (Wiebe, 2007). Los huevos más asimétricos de la gaviota concuerdan con su nidificación en el suelo, en ligeras depresiones, donde tienen más probabilidades de que, por el viento o por la propia actividad de los padres, rueden fuera del nido y la forma elongada disminuye el arco de rodamiento, minimizando este peligro. En estas condiciones, también están sometidos a mayores variaciones térmicas, lo que pudiera influir en la selección natural de la proporción volumen-superficie. Dives atroviolaceus es una especie que construye un nido de ramas, cóncavo, en el cual el riesgo de caída es mínimo y no existe problemas fuertes con la temperatura ni el oxígeno. En el trabajo de Preston (1969) la caracterización de los huevos de 33 especies de Laridae resultó en un índice de elongación medio de 1.40 y de asimetría de 0.264 , ligeramente superior en ambos casos al de los Icteridae (19 especies analizadas), de 1.38 y 0.153 , respectivamente. En este estudio no se incluyó ningún psitácido, pero otros grupos de nidificantes en cavidades, como Picidae y Strigidae, tuvieron índices de elongación y asimetría mucho menores.

El método propuesto, los descriptores elípticos de Fourier, a pesar de su relativa complejidad matemática, está totalmente automatizado con lo cual los errores humanos en las mediciones se minimizan. No emplea vías complejas para la adquisición de los datos (equipos "delineadores") sino solo una imagen digital, con un procesamiento mínimo. Su capacidad de reconstruir las formas automáticamente, luego de los procesamientos estadísticos, es también atractiva y no requiere habilidades de dibujo del investigador permitiendo identificar visualmente la ubicación de las diferencias detectadas. Además, en principio, utiliza un enfoque holístico de la verdadera forma, independientemente de las dimensiones y deja abierta una vía 
para numerosos estudios de patrones de variación, fenómenos ecológicos y herencia de las características del huevo. Probablemente, un análisis más profundo de un mayor número de especies de aves revele otras relaciones entre la forma del huevo y las características de las estrategias reproductivas.

\section{AGRADECIMIENTOS}

El autor desea agradecer a la dirección del Instituto de Ecología y Sistemática, y a los curadores de la colección de huevos, por permitir su empleo en este estudio, y a los revisores anónimos por sus oportunos señalamientos al manuscrito.

\section{RESUMEN}

La forma de los huevos es difícil de cuantificar por la ausencia de fórmulas exactas que describan su geometría. Se describe un algoritmo para la caracterización y comparación de estas formas, basado en la aplicación de las funciones de Fourier. Estas permiten delinear cualquier tipo de contorno cerrado y han sido aplicadas efectivamente al análisis de varias formas biológicas. Se describen los pasos para la toma de datos, su procesamiento y el empleo del programa SHAPE para la obtención de los descriptores, a partir de un estudio de caso. En este se comparan las formas de los huevos de tres especies de aves que representan tres estrategias reproductivas bien diferentes: el Catey (Aratinga euops), la Gaviota Real (Thalasseus maximus) y el Totí (Dives atroviolaceus). A partir de 73 fotografías digitales a huevos depositados en colecciones, se calcularon los coeficientes de las funciones para 4,6 , 8, 16 y 20 armónicos, y se redujeron por medio de un Análisis de Componentes Principales. Los puntajes de los componentes que describen hasta el $90 \%$ de la variabilidad fueron empleados en un Análisis de Función Discriminante Lineal para analizar la posibilidad de separar los huevos según sus formas. Con solo cuatro armónicos los primeros cinco componentes explicaron $97 \%$ de la varianza en formas. Más armónicos disminuyen la varianza explicada, requiriéndose hasta ocho componentes para explicar la misma cantidad. Los polígonos convexos en el espacio discriminante muestran una clara distinción entre especies sugiriendo la posible discriminación (errores de clasificación entre 7-15\%). Los errores en las clasificaciones estuvieron relacionados a diferencias específicas en la forma entre especies. En el estudio de caso, los huevos de $A$. euops fueron perfectamente clasificados pero en las otras especies los errores fueron entre 5 y $29 \%$, con este número de armónicos y componentes empleados. El algoritmo propuesto a pesar de su complejidad matemática aparente muestra muchas ventajas para cuantificar las formas de los huevos permitiendo una mayor comprensión de los factores que determinan esta variable en las aves.

Palabras clave: forma del huevo, descriptores de Fourier, EFD, programa SHAPE.

\section{REFERENCIAS}

Andrade, I. M., Mayo, S. J., Kirkup, D., \& Van den Berg, C. (2010). Elliptic Fourier analysis of leaf outline shape in forest fragment populations of Anthurium sinuatum and $A$. pentaphyllum (Araceae) from northeast Brazil. Kew Bulletin, 65, 1-18.

Adams, D. C., Rohlf, F. J., \& Slice, D. E. (2004). Geometric morphometrics: ten years of progress following the 'revolution'. Italian Journal of Zoology, 71, 5-16.

Barta, Z. \& Székely, T. (1997). The optimal shape of avian eggs. Functional Ecology, 11, 656-662.

Briskie, J. V. \& Sealy, S. G. (1990). Variation in size and shape of least flycatcher eggs. Journal of Field Ornithology, 61(2), 180-191.

Carey, C. (1996). Avian energetic and nutritional ecology. New York: Ed. Chapman \& Hall.

Coulson, J. C., Potts, G. R., \& Horobin, J. (1969). Variation in the eggs of the Shag (Phalacrocorax aristotelis). Auk, 86(2), 232-245.

Denis, D. (2002). Ecología reproductiva de siete especies de garzas (Aves: Ardeidae) en la ciénaga de Birma, Cuba. (Tesis de Doctorado). Universidad de La Habana, Cuba.

Denis, D. \& Olavarrieta, U. (2011). ¿Existe isomorfía en los huevos de las garzas (Aves: Ardeidae)? Animal Biodiversity and Conservation, 34.1, 35-45.

Ferson, S., Rohlf, F. J., \& Koehn, R. K. (1985). Measuring shape variation of twodimensional outlines. Systematic Zoology, 34, 59-68.

Freeman, H. (1974). Computer processing of line drawing images. Computing. Surveys, 6, 57-97.

Furuta, N., Ninomiya, S., Takahashi, S., Ohmori, H., \& Ukai, Y. (1995). Quantitative evaluation of soybean (Glycine $\max$ L. Merr.) leaflet shape by principal component scores based on elliptic Fourier descriptor. Breeding Science, 45, 315-320.

Gemperle, M. E. \& Preston, F. W. (1955). Variation of shape in the eggs of the common tern in the clutchsequence. Auk, 72, 184-198. 
Gill, F. B. (1998). Ornithology. Nueva York: Ed. Freeman \& Co.

Grant, P. R. (1982). Variation in the size and shape of darwin's finch eggs. Auk, 99, 15-23.

Hoyt, D. F. (1976). The effect of shape on the surface-volume relationships of birds' eggs. Condor, 78, 343-349.

Iwata, H., Nesumi, H., Ninomiya, S., Takano, Y., \& Ukai, Y. (2002) Diallel Analysis of Leaf Shape Variations of Citrus Varieties Based on Elliptic Fourier Descriptors. Breeding Science, 52, 89-94.

Iwata, H. \& Ukai, Y. (2002). SHAPE: A computer program package for quantitative evaluation of biological shapes based on elliptic Fourier descriptors. Journal of Heredity, 93, 384-385.

Janiga, M. (1997). Effects of geographic variation and hatching asynchrony on size and shape of eggs of the feral pigeon (Columbia livia). Folia Zoológica, 46(1), 23-32.

Jover, L., Ruiz, X., \& González-Martín, M. (1993). Significance of intraclutch egg size variation in the Purple Heron. Ornis Scandinavica, 24(2), 127-134.

Kendall, D. (1977). The diffusion of shape. Advances in Applied Probability, 9, 428-430.

Kendeigh, S. C. (1975). Effects of parentage on egg characteristics. Auk, 92, 163-164.

Kincaid, D. T. \& Schneider, R. B. (1983). Quantification of leaf shape with a microcomputer and Fourier transformation. Canadian Journal of Botany, 61, 2333-2342.

Kim, S. J., Kim, B. W., \& Kim, D. P. (2011). Tree recognition for landscape using by combination of features of its leaf, flower and bark. Proceedings of SICE Annual Conference (SICE), 1147-1151.

Kuhl, F. P. \& Giardina, C. R. (1982). Elliptic Fourier features of a closed contour. Computer Graphics and Image Processing, 18, 236-258.

Lack, D. (1947). Darwin's finches. Cambridge: Cambridge Univ. Press.

Lonn, M. \& Prentice, H. C. (1995). The structure of allozyme and leaf shape variation in isolated, range-margin populations of the shrub Hippocrepis emerus (Leguminosae). Ecography, 18, 276-285.

Mand, R., Nigul, A., \& Sein, E. (1986). Oomorphologya: new method. Auk, 103, 613-617.

Martin, T. E., Bassar, R. D., Bassar, S. K., Fontaine, J. J., Lloyd, P., Mathewson, H. A., Niklison, A. M., \& Chalfoun, A. (2006). Life-history and ecological correlates of geographic variation in egg and clutch mass among passerine species. Evolution, 60(2), 390-398.

McLellan, T. \& Endler, J. A. (1998). The relative success of some methods for measuring and describing the shape of complex objects. Systematic Biology, 47, 264-281.

Neto, J. C., Meyer, G. E., Jones, D. D., \& Samal, A. K. (2006). Plant species identification using Elliptic Fourier leaf shape analysis. Computers and Electronics in Agriculture, 50, 121-134.

Ojanen, M., Orell, M., \& Vaisinen, R. A. (1979). Role of heredity in egg size variation in the Great Tit Parus major and the Pied Flycatcher Ficedula hypoleuca. Ornis Scandinavica, 10, 22-28.

Orr, R. T. (1945). A study of captive Galapagos finches of the genus Geospiza. Condor, 47, 177-201.

Otto, C. (1979). Environmental factors affecting egg weight within and between colonies of Fieldfare Turdus pilaris. Ornis Scandinavica, 10, 111-116.

Petersen, M. R. (1992). Intraspecific variation in egg shape among individual emperor geese. Journal of Field Ornithology, 63(3), 344-354.

Preston, F. W. (1968). The shapes of birds' eggs: mathematical aspects. $A u k, 85,454-463$.

Preston, F. W. (1969). Shapes of birds' eggs: extant North American families. Auk, 86, 246-264.

Preston, F. W. (1974). The volume of an egg. Auk, 91,132-138.

Preston, F. W. \& Preston, E. J. (1953). Variation of birds' eggs within the clutch. Annals of Carnegie Museum, $33,129-139$

Preston, F. W. (1953). The shapes of birds' eggs. Auk, 70, 160-182.

Ricklefs, R. E. (1984). Variation in the size and composition of eggs of the European Starling. Condor, 86, $1-6$.

Rohlf, F. J. \& Archie, J. W. (1984). A comparison of Fourier methods for the description of wing shape in mosquitoes (Diptera: Culicidae). Systematic Zoology, 33, 302-317.

Singh, K., Gupta, I., \& Gupta, S. (2013). Classification of Bamboo Species by Fourier and Legendre Moment. International Journal of Advanced Science and Technology, 50, 61-70.

Svensson, B. W. (1978). Clutch dimensions and aspects of the breeding strategy of the Chaffinch Fringilla coelebs in northern Europe: a study based on egg collections. Ornis Scandinavica, 9, 66-83. 
Vaisinen, R. A., Hilden, O., Soikkeli, M., \& Vuolanto, S. (1972). Egg dimension variation in five wader species: the role of heredity. Ornis Fennica, 49, 25-44.

Van Noordwijk, A. J., Keizer, L. C. P., Van Balen, J. H., \& Scharloo, W. (1981). Genetic variation in egg dimensions in natural populations of the Great Tit. Genetica, 55, 221-232.

White, R. J. \& Prentice, H. C. (1988). Comparison of shape description methods for biological outlines. In H. H. Bock (Ed.), Classification and Related Methods of
Data Analysis (pp. 395-402). Amsterdam: Elsevier Sci. Pub.

Wiebe, K. L. (2007). Hypoxia probably does not explain short incubation periods of woodpeckers. Condor, 109(4), 976-979.

Zhan, Q. B. \& Wang, X. L. (2012). Elliptic Fourier Analysis of the Wing Outline Shape of Five Species of Antlion (Neuroptera: Myrmeleontidae: Myrmeleontini). Zoological Studies, 51(3), 399-405. 